\title{
Identification variétale des orges par électrophorèse. Description de 280 variétés
}

\author{
$\mathrm{J}$ Lallemand, $\mathrm{F}$ Briand \\ INRA/GEVES, Domaine du Magneraud, 17700 Surgères, France
}

(Reçu le 23 mars 1990, accepté le 18 mai 1990)

\begin{abstract}
Résumé - Les diagrammes protéiques de 280 variétés d'orge ont été décrits. La technique d'électrophorèse sur gel d'acrylamide contenant du SDS permet de séparer les variétés en 86 groupes. En utilisant en plus les diagrammes des estérases et des phosphatases acides, il est possible d'établir une clé d'identification pour les 12 orges d'hiver et 13 orges de printemps les plus cultivées en France.
\end{abstract}

orge / enzyme / protéine / électrophorèse / identification variétale

Summary - Identification of barley varieties using electrophoresis. Description of 280 varieties. Two hundred and eighty varieties of barley are described for their seed protein patterns using SDS-PAGE. This technique enables us to make 86 groups. By the additional use of esterases and acid phosphatases, it is possible to make an identification key for the 12 winter varieties and 13 spring varieties most used in France.

barley / enzyme / protein / electrophoresis / varietal identification

\section{INTRODUCTION}

Les variétés d'orge inscrites au catalogue officiel sont de plus en plus nombreuses ( 183 au catalogue français en 1987) et deviennent de plus en plus difficiles à distinguer les unes des autres. Les critères morphologiques et physiologiques traditionnels pour la distinction ne suffisent pas toujours. Ils présentent, de plus, l'inconvénient d'être dépendants de l'environnement et parfois assez subjectifs (couleur ou largeur de feuilles, par exemple), ce qui nécessite pour une étude fiable, la présence de très nombreux témoins. Un autre inconvénient est l'exigence en espace et en temps.

L'électrophorèse des protéines de réserve et des enzymes offre la possibilité d'aller plus loin dans la distinction de façon rapide ( 1 jour) et fiable (indépendance vis-à-vis du milieu, répétabilité).

Nous présentons ici les résultats d'électrophorèse obtenus sur une collection variétale de 280 orges d'hiver et de printemps.

\section{MATÉRIEL ET MÉTHODE}

\section{Matériel}

La collection variétale étudiée pour le polymorphisme des protéines de réserve comprend 280 variétés incluant toutes celles inscrites au catalogue officiel français de 1988.

Pour le polymorphisme enzymatique, seules sont décrites ici les 12 variétés d'orge d'hiver et les 13 variétés d'orge de printemps les plus cultivées en France.

\section{Choix de la méthode}

\section{Électrophorèse des protéines}

De nombreuses méthodes ont été décrites pour l'électrophorèse des protéines de l'orge. Deux tendances principales existent : l'utilisation de gel contenant du SDS, ou de gels acides contenant de l'urée (tableau I). Kapala (1981) associe l'urée au système SDS. La méthode officielle de I'ISTA (International 
Tableau I. Utilisation des techniques d'électrophorèse de protéines de réserve de l'orge par différents auteurs.

\begin{tabular}{llll}
\hline \multicolumn{1}{c}{$S D S$} & & & Urée \\
& & & \\
Montembault & 1982 & Marchylo et La Berge & $1981 \mathrm{a}, \mathrm{b}$ \\
Smith et Payne & 1984 & Laurière et Mossé & 1982 \\
Bunce et al & 1984 & Schildbach et Burbidge & 1984 \\
Heisel et al & 1986 & Cooke et Morgan & 1986 \\
Nielsen et Johansen & 1986 & Gebre et al & 1986 \\
Marchylo & 1987 & & \\
\hline
\end{tabular}

Seed Testing Association) inspirée de Schildbach et Burbidge (1984) et proposée par Cooke et Morgan (1986) utilise des gels acides contenant de l'urée.

Nous avons adopté la méthode SDS décrite par Montembault (1982) qui nous semble donner une meilleure séparation que la méthode ISTA. De plus, cette méthode est, à peu de choses près, identique à celle déjà utilisée au laboratoire pour le blé dur et qui est la méthode officielle de I'IRTAC (Institut de recherches technologiques et agro-alimentaires des céréales).

Dans cette méthode, les protéines sont extraites dans du tampon TRIS $\mathrm{HCl}(0,06 \mathrm{~mol} / \mathrm{l}, \mathrm{pH} 6,8)$ contenant $2 \%$ de SDS, $0,01 \%$ de pyronine, $10 \%$ de glycérol, $8 \%$ de mercapto-éthanol et $15 \%$ de DMF. La séparation des protéines se fait dans un gel d'acrylamide $(180 \times 160 \times 1,5 \mathrm{~mm})$ dans un système discontinu de tampons (tableau II). L'électrophorèse est faite à ampérage constant de $24 \mathrm{~mA}$ pendant $16 \mathrm{~h}$.

\section{Électrophorèse des enzymes}

Pour ce qui est des enzymes, nous avons choisi les systèmes suivants : estérases et phosphatases

Tableau II. Solutions utilisées pour l'électrophorèse.

$\begin{array}{ccc}\text { Gelde } & \text { Gelde } & \text { Tampon } \\ \text { concentration séparation } & \text { bac } \\ \text { à 3,5\% } & \text { à } 13 \% & \end{array}$

\begin{tabular}{|c|c|c|c|}
\hline \multicolumn{4}{|l|}{ Acrylamide } \\
\hline $51,98 \%$ & $1,35 \mathrm{ml}$ & $20 \mathrm{ml}$ & - \\
\hline $\begin{array}{l}\text { Bis } 0,32 \% \\
\text { TRIS } 1 \mathrm{~mol} / 1\end{array}$ & $3,17 \mathrm{ml}$ & $26 \mathrm{ml}$ & - \\
\hline $\begin{array}{l}\mathrm{pH} 6,8 \\
\text { TRIS } 1 \mathrm{~mol} / \mathrm{l}\end{array}$ & $2,5 \mathrm{ml}$ & - & - \\
\hline $\begin{array}{c}\mathrm{pH} 8,8 \\
\text { Persulfate }\end{array}$ & - & $30 \mathrm{ml}$ & - \\
\hline d'ammonium $1 \%$ & $0,875 \mathrm{ml}$ & $2 \mathrm{ml}$ & - \\
\hline SDS $10 \%$ & $0,233 \mathrm{ml}$ & $0,8 \mathrm{ml}$ & $5 \mathrm{~g}(0,1 \%)$ \\
\hline TEMED & $17,5 \quad \mu \mathrm{l}$ & $40 \mu 1$ & - \\
\hline $\mathrm{H}_{2} \mathrm{O}$ & $12,3 \mathrm{ml}$ & - & qsp 51 \\
\hline TR̂IS & - & - & $15 \mathrm{~g}$ \\
\hline Glycine & - & - & $70,55 \mathrm{~g}$ \\
\hline
\end{tabular}

acides. En effet, sur la collection variétale étudiée, ce sont eux qui montraient le plus grand polymorphisme. Le protocole adopté est celui de Nielsen et Johansen (1986). Le tableau IV donne les correspondances entre notre nomenclature et celle de Nielsen et Johansen.

\section{Interprétation des résultats}

Après coloration des gels, on observe les protéines réparties en 4 zones notées A, B, C, D (fig 1). La zone A n'est pas interprétée. Les autres zones peuvent se présenter sous différentes formes caractéristiques de la variété : 5 pour la zone $D$, une vingtaine pour les zones $B$ et $C$. Les formes les plus courantes sont représentées sur la figure 2. Pour les zones $B$ et $C$, les types sont numérotés de 1 à 19. Pour la zone $D$, les types différents sont : $N, L 1, L 2, H 1, H 2-N$ étant de loin le plus fréquent. Une variété possédant le type 1 dans la zone $\mathrm{B}, 2$ dans la zone $\mathrm{C}$ et $\mathrm{H} 1$ dans la zone $\mathrm{D}$, sera notée : $\mathrm{B} 1 \mathrm{C} 2 \mathrm{H} 1$. La variété en $5^{\mathrm{e}}$ position sur

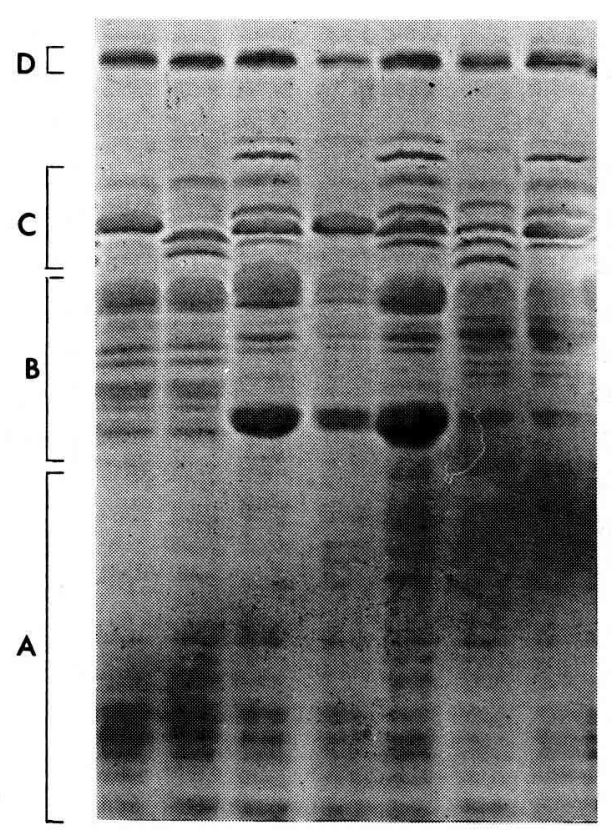

Fig 1. Migration des protéines de réserve d'orge dans un gel contenant du SDS. Les types présents sont de gauche à droite : $B 2 C 5 N, B 10 C 1 N, B 10 C 6 N, B 1 C 1 N, B 8 C 8 N, B 8 C 2 N$. 


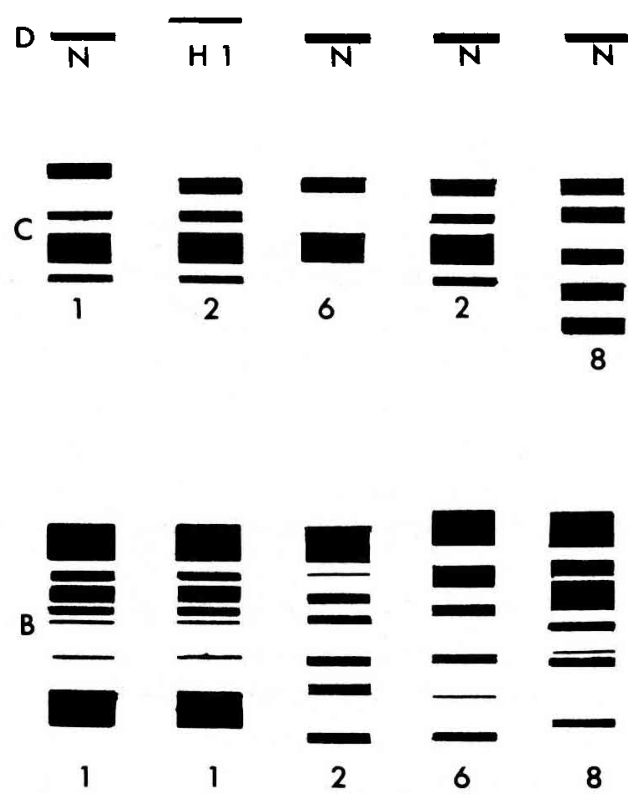

Fig 2. Représentation schématique des électrophorégrammes observés le plus fréquemment dans la collection d'orges étudiée.

la figure 1 sera notée : $\mathrm{B} 8 \mathrm{C} 8 \mathrm{~N}$. Nous avons gardé la numérotation de Montembault (1982) pour tous les types qu'il a déjà décrits.

Six types supplémentaires ont été trouvés pour la zone $B, 6$ pour la zone $C$ et 1 pour la zone $D$. Les variétés représentatives de ces nouveaux types sont indiquées dans le tableau III.

Pour les enzymes, nous avons choisi une numérotation classique, l'allèle codant l'enzyme de mobilité la plus élevée étant appelé $a$, le suivant $b$, etc. Les allèles nuls ont été notés $\mathrm{N}$. Nous donnons sur le tableau IV la correspondance entre cette notation et celle de Nielsen et Johansen (1988).

Tableau III. Variétés représentatives des nouveaux types trouvés.

\begin{tabular}{ll}
\hline Type & Variété \\
\hline & \\
B 14 & Hermine \\
B 15 & Nackta \\
B 16 & Cabro \\
B 17 & Vista \\
B 18 & Sigma \\
B 19 & Alouette \\
C 14 & Apache \\
C 15 & Firecrest \\
C 16 & Capri \\
C 17 & Norka \\
C 18 & Goélette \\
C 19 & Thérèse \\
L 2 & Nackta \\
\hline
\end{tabular}

Tableau IV. Correspondances entre les notations utilisées pour ce travail (1) et celles de Nielsen et Johansen (1988) (2).

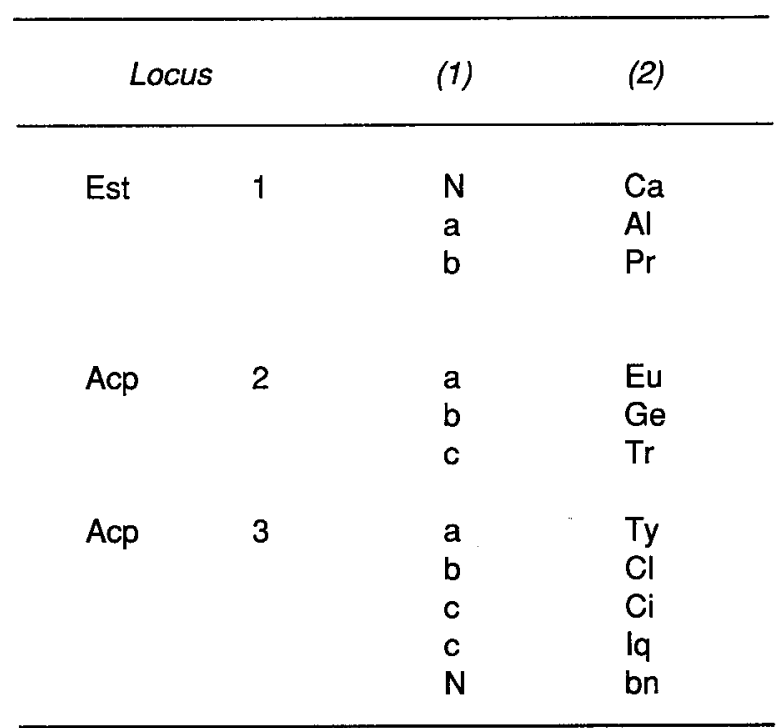

\section{RÉSULTATS}

Les résultats de l'électrophorèse des protéines obtenus sur les 280 variétés d'orge étudiées ont été publiés dans un rapport interne et peuvent être obtenus des auteurs. Ces résultats permettent de répartir les variétés en 86 groupes différents. Seules 45 variétés se distinguent de toutes les autres. Les variétés restantes se répartissent comme suit : 15 groupes de 2 variétés, 8 groupes de 3 variétés, 3 groupes de 4 variétés, 3 groupes de 5 variétés, 1 groupe de 6 variétés, 1 groupe de 7 variétés, 1 groupe de 9 variétés, 1 groupe de 10 variétés, 2 groupes de 11 variétés, 1 groupe de 13 variétés, 1 groupe de 15 variétés, 1 groupe de 16 variétés, 1 groupe de 18 variétés, 1 groupe de 38 variétés.

Certains groupes sont particulièrement représentés. Par exemple B8C8N chez les orges d'hiver. Ceci est dû à l'usage plus particulier de certaines variétés comme géniteurs.

Ces résultats montrent que les diagrammes protéiques ne suffisent pas pour l'identification des variétés. C'est pourquoi la description enzymatique a été entreprise. Pour les enzymes, nous nous sommes contentés d'étudier 25 variétés (les 12 orges d'hiver et les 13 orges de printemps les plus cultivées en France). Ces résultats combinés à ceux de l'électrophorèse des protéines permettent d'établir pour ces variétés une clé de détermination (tableau V). Seules 2 variétés, Sonja et Flamenco, ne sont pas distinguées. Ceci s'explique facilement par le fait que 
Tableau V. Clé de détermination des 12 variétés d'hiver et 13 variétés de printemps les plus cultivées.

\begin{tabular}{|c|c|c|c|}
\hline \multirow{2}{*}{$\mathrm{B} 1 \mathrm{C} 1 \mathrm{~N}$} & ACP $3: B$ & & Menuet \\
\hline & $\mathrm{ACP} 3: \mathrm{C}$ & & Golf \\
\hline B1C5 N & & & Madelon \\
\hline $\mathrm{B} 1 \mathrm{C} 6 \mathrm{~N}$ & ACP $3: B$ & & Kym \\
\hline & ACP $3: C$ & & Cameo \\
\hline $\mathrm{B} 1 \mathrm{C} 7 \mathrm{~N}$ & & & Klaxon \\
\hline $\mathrm{B} 1 \mathrm{C} 13 \mathrm{~N}$ & & & Panda \\
\hline $\mathrm{B} 2 \mathrm{C} 5 \mathrm{~N}$ & $\begin{array}{l}\text { ACP } 2: A \\
\text { ACP } 2: C\end{array}$ & & $\begin{array}{l}\text { Aramir } \\
\text { Natasha } \\
\text { Triumph }\end{array}$ \\
\hline B3C1 N & & & \\
\hline $\mathrm{B} 6 \mathrm{C} 2 \mathrm{~N}$ & $\begin{array}{l}\text { ACP } 2: A \\
\text { ACP } 2: C\end{array}$ & EST $1: B$ & $\begin{array}{l}\text { Cérès } \\
\text { Magie }\end{array}$ \\
\hline & & EST $1: \mathrm{N}$ & Alpha \\
\hline $\mathrm{B} 6 \mathrm{C} 2 \mathrm{H}$ & & & Celtic \\
\hline $\mathrm{B} 6 \mathrm{C} 16 \mathrm{H}$ & & & Capri \\
\hline $\mathrm{B} 7 \mathrm{C} 2 \mathrm{~N}$ & $\begin{array}{l}\text { ACP } 2: A \\
A C P 2: C\end{array}$ & ACP $3: B$ & $\begin{array}{l}\text { Delta } \\
\text { Bérénice }\end{array}$ \\
\hline & & ACP $3: C$ & Béatrice \\
\hline $\mathrm{B} 8 \mathrm{C} 2 \mathrm{~N}$ & EST 1 : B & & Express \\
\hline & EST $1: N$ & & Plaisant \\
\hline $\mathrm{B} 8 \mathrm{C} 8 \mathrm{~N}$ & ACP $2: A$ & & Sonja ou Flamenco \\
\hline & $\mathrm{ACP} 2: \mathrm{C}$ & & Igri \\
\hline $\mathrm{B8C8} \mathrm{H}$ & & & Smash \\
\hline $\mathrm{B} 13 \mathrm{C} 12 \mathrm{~N}$ & & & Barberousse \\
\hline
\end{tabular}

ces 2 variétés sont étroitement apparentées, Flamenco résultant du croisement de France Dea par Sonja. Toutes les autres peuvent être identifiées de façon unique.

\section{CONCLUSION}

En conclusion, l'électrophorèse est un bon outil pour la distinction variétale. Elle permet de distinguer les variétés les plus couramment utilisées, ce qui rend possible les applications suivantes : contrôle d'identité de lots; contrôle de pureté des semences certifiées; aide aux tests officiels de distinction pour les variétés candidates à l'inscription au catalogue.

\section{RÉFÉRENCES}

Bunce N, Shewry PR, Miflin BJ (1984) The identification of barley cultivars by analysis of hordein polypeptides. Proc ISTA Symp Biochem tests for Cultivar Identification, 69-77

Cooke RJ, Morgan AG (1986) A revised classification of barley cultivars using a standard reference electrophoresis method. I Natl Inst Agric Bot 17, 169178

Gebre H, Khan K, Foster AE (1986) Barley Cultivar Identification by polyacrylamide gel electrophoresis of hordein proteins. Crop Sci 26, 454-460

Heisel SE, Peterson DM, Jones BL (1986) Identification of United States Barley Cultivars by Sodium Dodecyl Sulfate Polyacrylamide Gel Electrophoresis of Hordeins. Cereal Chem 63, 500-505

Kapala A (1981) Variability of electrophoretic subunit patterns of hordein proteins in spring barley. Genet Pol 22, 159-177

Laurière M, Mossé J (1982) Polyacrylamide Gel-Urea Electrophoresis of Cereal Prolamins at Acidic pH. Anal Biochem 122, 20-25

Marchylo BA (1987) Barley cultivar identification by SDS gradient PAGE analysis of hordein. Can $J$ Plant Sci 67, 927-944

Marchylo BA, La Berge DE (1981a) Barley cultivar identification by electrophoretic analysis of hordein proteins. I. Extraction and separation of hordein proteins and environmental effects of the hordein electrophoregram. Can J Plant Sci 60, 1343-1350

Marchylo BA, La Berge DE (1981b) Barley cultivar identification by electrophoretic analysis of hordein proteins. II. Catalogue of electrophoregram formulae for canadian-grown barley cultivars. Can J Plant Sci $61,859-870$

Montembault A (1982) Étude biochimique et génétique des protéines de l'orge. Thèse $3^{e}$ cycle, Montpellier

Nielsen G, Johansen HB (1986) Proposal for the identification of barley varieties based on the genotypes for 2 hordein and 39 isoenzyme loci of 47 reference varieties. Euphytica 35, 717-728

Schildbach R, Burbidge MJ (1984) Effects of electrophoretic quality control on the purity of commercial shipments of barley and malt. Proc ISTA Symp biochem tests for Cultivar Identification, 60-68

Smith DB, Payne PI (1984) A procedure for the routine determination of electrophoretic band patterns of barley and malt endosperm proteins. $J$ Natl Inst $A g$ ric Bot $16,487-498$ 